\title{
Incorporación del estudio de ADN fetal en sangre materna al cribado de cromosomopatías
}

\author{
Beatriz Rojas PE. ${ }^{1}$, Isabel González B. ${ }^{1}$, Amado Tapia L. ${ }^{2}$, Marta Lalana G. ${ }^{2}$, Lorena \\ Guardia D. ${ }^{1}$, Teresa Arribas M. ${ }^{1}$, María Ángeles Aragón S. ${ }^{1}$, Belén Carazo H. ${ }^{3}$ \\ 1 Servicio de Ginecología y Obstetricia, Hospital de Barbastro. ${ }^{2}$ Servicio de Análisis Clínicos, Hospital de Barbastro. \\ 3 Servicio de Ginecología y Obstetricia, Hospital Clínico Universitario Lozano Blesa, Zaragoza. España.
}

\section{RESUMEN}

Objetivo: Evaluar la efectividad del cribado combinado de primer trimestre para la detección prenatal de aneuploidías tras 6 años de implantación en nuestro servicio y su repercusión en la disminución de pruebas diagnósticas invasivas. Se propone establecer un protocolo para incorporar el estudio de ADN fetal en sangre materna a partir de las revisiones bibliográficas publicadas. Método: Se evaluó el riesgo de anomalía cromosómica fetal en 3177 gestaciones mediante cribado combinado de primer trimestre entre enero de 2011 y diciembre de 2014. Se revisaron las amniocentesis realizadas desde que se instauró el cribado combinado en 2008 comparándolas con las de los 5 años anteriores. Resultados: La tasa de detección del cribado para trisomía 21 fue del 94,4\% y la tasa de falsos positivos de 6,4\%. En el año 2005 estábamos realizando 194 amniocentesis, tras 6 años de implantación del cribado, en el año 2013 se realizaron 35 amniocentesis lo que implica una disminución del $70 \%$. Conclusiones: El cribado combinado de primer trimestre ha demostrado una mayor tasa de detección para trisomía 21 que el cribado de segundo trimestre y/o la edad materna, además de que ha llevado a una importante reducción en el número de pruebas invasivas. En los próximos años la incorporación del estudio de ADN fetal mejorará la detección de aneuploidías, con una drástica disminución de las pruebas invasivas por lo que se hace necesario la implantación de nuevos protocolos.

\section{PALABRAS CLAVE: Aneuploidía, cribado combinado, sonograma genético, diagnóstico prenatal no invasivo}

\section{SUMMARY}

Aims: To evaluate the effectiveness of first trimester combined screening in the prenatal detection of aneuploidy after 6 years of implantation in our service and its impact in reducing invasive diagnostic tests. It is proposed to establish a protocol to incorporate the study of fetal DNA in maternal blood from published literature reviews. Methods: The risk of fetal chromosomal anomalies was assessed in 3177 pregnancies with first trimester combined screening between January 2009 and December 2014. The amniocenteses performed were checked against those of the previous 5 years. Results: The detection rate of screening for trisomy 21 was $94.4 \%$ and the false-positive rate was $6.4 \%$. In 2005 there were 194 amniocenteses. In 2013, 5 years after the introduction of screening, 68 amniocenteses were performed, representing a $70 \%$ reduction in invasive procedures. Conclusions: First trimester combined screening has shown a higher detection rate for trisomy 21 that the second trimester screening and/or maternal age, and has substantially reduced the use of invasive prenatal diagnostics procedures. In the coming years, the incorporation of the study of fetal DNA improve the detection of aneuploidys with a drastic reduction of invasive tests so that, the implementation of new protocols is necessary.

KEY WORDS: Aneuploidy, combined screening, genetic sonogram, non-invasive prenatal diagnosis 


\section{INTRODUCCIÓN}

El primer método de cribado para la trisomía 21, introducido en la década de los 70, fue la edad materna avanzada. Se definió el grupo de "alto riesgo" como las mujeres de un mínimo de 35 años, lo que constituía el $5 \%$ de la población embarazada (1). El aumento en la proporción de gestantes de más de 35 años, junto con la necesidad de un procedimiento invasivo para la obtención del cariotipo y que conlleva riesgo de pérdida fetal, ha llevado a la investigación de métodos de cribado más eficaces.

En los años 90, se introdujo el cribado mediante la combinación de la edad materna y el grosor de la translucencia nucal (TN) a las $11-13^{6}$ semanas de gestación, estudiado sobre todo por el grupo de la Fetal Medicine Foundation liderado por K. Nicolaides (2). Éste método ha demostrado ser capaz de identificar el $75 \%$ de los fetos afectados, con una tasa de falsos positivos del $5 \%$. Posteriormente, la edad materna se ha combinado con la TN y la bioquímica sérica materna: Hormona Gonadotropina Coriónica Humana libre (HCG), y la proteína plasmática A asociada al embarazo (PAPP-A) para identificar en el primer trimestre al $85-90 \%$ de los fetos afectados (1). Hasta que se incorpore el estudio del ADN fetal en sangre materna al cribado de primer trimestre en nuestro medio, éste es de momento el método de cribado más eficaz, además de ser universal y poder aplicarse a todas las pacientes independientemente de su edad.

El objetivo de este estudio es conocer los resultados del cribado combinado en el primer trimestre de gestación tras 5 años de implantación en nuestro medio y su repercusión en la disminución de técnicas invasivas, además de proponer un protocolo de aplicación del estudio de ADN fetal como un paso más en el diagnóstico prenatal no invasivo.

\section{SUJETOS Y MÉTODOS}

Se han analizado 3177 cribados bioquímicos de primer y segundo trimestre realizados entre el 1 de enero de 2011 y el 31 de diciembre de 2014, realizados en población general no seleccionada.

El cribado combinado de primer trimestre se puso en marcha en junio de 2008, para un área que abarca una población de 100000 habitantes, atendiéndose en nuestro hospital unos 700 partos al año. Se ofrece a todas las gestantes que acuden a la primera visita de embarazo entre las $11-13^{6}$ semanas o, lo que es lo mismo, cuando la longitud craneocaudal (LCC) en esa primera ecografía está entre 45 y $85 \mathrm{~mm}$. Los criterios de exclusión son embarazo múltiple de más de dos fetos y la revocación por parte de la paciente, después de haber sido debidamente informada y suministrando consentimiento informado por escrito. Si la pacien- te no llega a realizarse cribado de primer trimestre pero está dentro de las primeras 18 semanas de gestación, se le ofrece la posibilidad de cribado bioquímico de segundo trimestre mediante doble test determinándose alfafetoproteína (AFP) y HCG o amniocentesis de entrada en el caso de pacientes de más de 38 años.

En los primeros años de implantación del cribado (2008 a 2010), se ofrecía amniocentesis a las embarazadas mayores de 38 años aunque el resultado del cribado de primer trimestre fuera de bajo riesgo, siguiendo la primera estrategia de cribado de Diagnóstico Prenatal que se había establecido en Cataluña en 1998 (3). También se seguía permitiendo la realización de técnicas invasivas a todas aquellas gestantes que la pidieran independientemente de su riesgo, lo que considerábamos como criterio "ansiedad materna". Esto hizo que la disminución en el número de pruebas invasivas fuera paulatina y que en esos años cambiaran los principales motivos de indicación. En 2011, siguiendo la Estrategia de Cribado de Cromosomopatías en Aragón y los protocolos que se estaban llevando a cabo en otras comunidades autónomas (3) se desestimaron los criterios "ansiedad materna" y edad materna como indicación de prueba invasiva (4).

El cribado se realiza en una sola visita. Se programa una exploración ecográfica entre las 11 y $13^{6}$ semanas, idealmente a las 12 , y tras ésta, se envía a la paciente a laboratorio para la extracción de sangre con los datos de la LCC y la TN, medidos según los criterios de la Fetal Foundation Medicine (5). Los valores obtenidos de PAPP-A, HCG y TN se convierten en múltiplos de la mediana (MoM) para la correspondiente edad gestacional y se calcula el riesgo. Se tienen en cuenta como factores de corrección el peso y la fecha de nacimiento de la paciente, el consumo de tabaco, la diabetes, la raza, si se trata de una fecundación in vitro y, en el caso de los gemelos, la corionicidad. El cálculo del riesgo de aneuploidías se envía en informe al obstetra que es el que informa a la paciente una semana después de la ecografía.

El punto de corte utilizado para ofrecer la realización de técnicas invasivas fue un riesgo igual o superior a 1/270 en el caso de trisomía 21 y 1/100 en el caso de trisomías 13 y 18. La obtención de cariotipo fetal se realizó mediante amniocentesis a partir de las 15 semanas.

Las pacientes con un riesgo menor de 1/270 continúan el seguimiento normal del embarazo y sólo se les ofrece amniocentesis si en la ecografía morfológica a las 20 semanas se observan malformaciones o marcadores menores de anomalías cromosómicas, corrigiendo su riesgo inicial obtenido por el cribado combinado con los likelihood ratio de los marcadores hallados, según protocolo de la Fetal Medicine Foundation. 


\section{RESULTADOS}

Durante el periodo estudiado se realizaron 3177 pruebas de tamizaje bioquímico, 3018 de ellas cribado combinado de primer trimestre y 159 doble test de segundo trimestre, en la mayoría de los casos inmigrantes que acuden tarde a primera visita de embarazo (Tabla I).

Las indicaciones de las amniocentesis desde 2007 se presentan en la Tabla II. Con la introducción del cribado combinado ha habido una total inversión de la indicación principal, además de que se han reducido el número de indicaciones hasta ser el cribado positivo prácticamente la única.

La tasa de detección para trisomías 13, 18 y 21 fue del $94,4 \%$, con el inconveniente de que en alguno de los casos diagnosticados en los primeros años del cribado no se llegó a realizar éste si ya había indicación de amniocentesis por otro motivo, por ejemplo translucencia (TN) mayor del P95. No obstante, se da por hecho, que en estos casos el resultado del cribado hubiera sido positivo por el alto valor de la TN. Considerando el punto de corte para la indicación de una prueba invasiva un riesgo mayor o igual a 270, la tasa de cribados positivos fue del $63 \%$. Si consideramos que el cribado bioquímico hubiera dado resultado positivo al introducir en el cálculo del mismo TN mayores del percentil 95, tras el cribado se hubieran detectado por cariotipo fetal 24 alteraciones cromosómicas graves (19 trisomías 21, una trisomía 13 , tres trisomías 18 y una 22).

Dos casos pasaron desapercibidos: una trisomía 21 en una paciente de 32 años y una trisomía 13 en una paciente de 33 años. En el recién nacido con trisomía 21 no se hallaron malformaciones ni durante el embarazo ni tras en el nacimiento, y en el caso de la trisomía 13 únicamente se vio un foco ecogénico de ventrículo izquierdo en la ecografía de nivel II y una aplasia cutis, además de una comunicación interventricular leve tras el nacimiento. La TN en el primero fue $1,5 \mathrm{~mm}$ y en el segundo $1,4 \mathrm{~mm}$ (Tabla III). La tasa de falsos positivos fue de $6,4 \%$.

Tabla I

CRIBADO COMBINADO. EVOLUCIÓN DURANTE LOS PRIMEROS 6 AÑOS DE CRIBADO, DATOS RECOGIDOS ÚNICAMENTE DESDE 2011

\begin{tabular}{lccccc}
\hline Año & 2011 & 2012 & 2013 & 2014 & Total \\
\hline Número cribados primer trimestre & 681 & 832 & 753 & 752 & 3177 \\
Riesgo >1/270 & 31 & 71 & 62 & 39 & 203 \\
Aneuploidías graves detectadas & 4 & 1 & 6 & 5 & 24 \\
Aneuploidías no detectadas & 1 & 100 & 100 & 0 & 2 \\
Tasa de detección (\%) & 80 & 8,1 & 7,9 & 4,9 & 94 \\
Tasa de falsos positivos (\%) & 4,3 & & & 6,4 \\
\hline
\end{tabular}

Tabla II

EVOLUCIÓN DE LAS INDICACIONES DE AMNIOCENTESIS DESDE 2007 A 2013

\begin{tabular}{lcccccccc}
\hline Año & 2007 & 2008 & 2009 & 2010 & 2011 & 2012 & 2013 & 2014 \\
\hline Edad materna (\%) & 72 & 68 & 37 & 49 & 40 & 26 & 18 & 20 \\
PAPP-A (\%) & 0 & 5 & 26 & 6 & 24 & 52 & 63 & 74 \\
Triple cribado (\%) & 16 & 11 & 3 & 7 & 3 & 6 & 9 & 0 \\
Ansiedad (\%) & 7 & 10 & 20 & 20 & 17 & 1 & 0 & 0 \\
Hallazgos ecográficos (\%) & 0 & 0 & 7 & 3 & 4 & 8 & 3 & 14 \\
Antecedentes (\%) & 4 & 2 & 4 & 0 & 3 & 3 & 2 & 2 \\
Total de amniocentesis & 200 & 124 & 101 & 96 & 76 & 81 & 68 & 35 \\
\hline
\end{tabular}

PAPP-A: cribado combinado de primer trimestre 
Tabla III

CROMOSOMOPATÍAS GRAVES EN NUESTRO MEDIO ENTRE 2009-2013

\begin{tabular}{|c|c|c|c|c|c|}
\hline Caso & Edad & Indicación & Screening & Cariotipo & Seguimiento \\
\hline 1 & 36 & Derrame pleural $15 \mathrm{~s}$ & No se hizo & $47 X Y+21$ & IVE \\
\hline 2 & 34 & $\mathrm{TN}$ & No se hizo & $47 X Y+21$ & IVE \\
\hline 3 & 39 & 39 años & No se hizo & $47 X X+21$ & IVE \\
\hline 4 & 32 & Sin diagnosticar & PAPP-A 1/422 & $47 X Y+21$ & RN vivo \\
\hline 5 & 38 & $\mathrm{TN}$ & No se hizo & $47 X Y+21$ & IVE \\
\hline 6 & 33 & $\mathrm{TN}$ & No se hizo & $47 X Y+21$ & IVE \\
\hline 7 & 29 & TN, canal AV & & $47 X X+21$ & RN vivo \\
\hline 8 & 43 & $\mathrm{TN}$ & No se hizo & $47 X X+21$ & IVE \\
\hline 9 & 38 & PAPP-A & PAPP-A 1/263 & $47 X Y+18$ & Muerte perinatal precoz \\
\hline 10 & 43 & $\mathrm{TN}$ & No se hizo & $47 X Y+21$ & RN vivo \\
\hline 11 & 25 & PAPP-A & PAPP-A 1/75 & $47 X Y+21$ & IVE \\
\hline 12 & 34 & $\mathrm{TN}$ & PAPP-A 1/5 & $47 X X+21$ & IVE \\
\hline 13 & 36 & TN, canal AV & No se hizo & $47 X Y+13$ & Aborto espontáneo \\
\hline 14 & 33 & Sin diagnosticar & PAPP-A 1/486 & $47 X X+13$ & Muerte perinatal precoz \\
\hline 15 & 45 & $\mathrm{TN}$ & No se hizo & $47 X X+21$ & IVE \\
\hline 16 & 35 & $\mathrm{TN}$ & PAPP-A 1/5 & $47 X X+21$ & IVE \\
\hline 17 & 39 & PAPP-A, ADN fetal (+) & PAPP-A 1/30 & $47 X Y+21$ & IVE \\
\hline 18 & 33 & TN, Ebstein & PAPP-A 1/5 & $47 X Y+22$ & IVE \\
\hline 19 & 40 & PAPP-A, mesomelia & PAPP-A 1/24 & $47 X Y+18$ & IVE \\
\hline 20 & 33 & $\mathrm{TN}$ & PAPP-A 1/5 & $47 X Y+18$ & Muerte fetal \\
\hline 21 & 43 & TN, canal AV & PAPP-A 1/5 & $47 X X+21$ & RN vivo \\
\hline 22 & 26 & PAPP-A & PAPP-A 1/94 & $47 X X+21$ & IVE \\
\hline 23 & 37 & $\mathrm{TN}$ & PAPP-A 1/5 & $47 X Y+21$ & IVE \\
\hline 24 & 36 & $\mathrm{TN}$ & PAPP-A 1/18 & $47 X Y+21$ & IVE \\
\hline 25 & 38 & $\mathrm{TN}$ & PAPP-A 1/5 & $47 X X+21$ & IVE \\
\hline 26 & 43 & PAPP-A & PAPP-A 1/11 & $47 X Y+21$ & RN vivo \\
\hline
\end{tabular}

IVE: Interrupción voluntaria del embarazo.

En los años previos a la implantación del cribado estábamos realizando aproximadamente 200 amniocentesis al año, la mayoría por edad materna para diagnosticar muy pocos casos por ese motivo. En el año 2013 se han realizado 68 amniocentesis, lo que supone un $66 \%$ menos. La tasa global de pérdida fetal es de $1,8 \%$ si consideramos los abortos de menos de 20 semanas ( 8 abortos en 5 años, 422 amniocentesis).

\section{DISCUSIÓN}

El cribado combinado para la detección de anomalías cromosómicas en el primer trimestre de la gestación, especialmente trisomía 21, es la estrategia recomendada en España y avalada por la Sociedad de Ginecología y Obstetricia $(5,6)$. En los últimos años se ha ido afianzando como el método de elección para el diagnóstico de aneuploidías, llegando ya al $94 \%$ de los centros encuestados en una revisión realizada a nivel nacional en 2011 que incluía al 30\% de las gestantes de toda España (7).

Históricamente la edad materna avanzada fue casi exclusivamente el único criterio para el cribado de cromosomopatías, lo que, junto con el retraso progresivo en la edad de maternidad, conducía a un aumento notable en el número de técnicas invasivas con el consiguiente aumento de pérdidas 
fetales inherentes a estas pruebas. La mayoría de las estrategias recomiendan únicamente tener en cuenta los resultados del cribado para decidir la realización de amniocentesis y en ningún caso la edad de la madre $(8,9)$, en nuestro centro hasta los años 2013-2014, todavía se les ofrecía la posibilidad de realizar prueba invasiva a las pacientes mayores de 40 años, por la posibilidad de otras cromosomopatías que no pueden sospecharse con el cribado combinado.

La introducción del cribado combinado y el progresivo abandono de la edad materna como criterio aislado para la indicación de técnicas invasivas junto con la erradicación del criterio "ansiedad materna”, ha conllevado la disminución del número de amniocentesis sin merma en la tasa de detección de síndrome de Down. En el año 2007 realizamos 100 amniocentesis para diagnosticar una anomalía cromosómica grave, mientras que en 2014 se realizaron 7 por cada una de ellas. Con el método de cribado combinado se obtiene una relación síndrome de Down confirmados/pérdida fetal de 2,2 frente al 0,9 que se obtiene con el test del segundo trimestre (10).

En nuestra población, la mediana de MoM en los cálculos de la TN es muy próxima a 1, aunque la medición de la misma es más observadordependiente que las determinaciones analíticas y requiere una curva de aprendizaje. Así se ha recogido en otros estudios de análisis de datos (11). El subestimar el valor de la TN repercutiría en una determinación de riesgos menores, que conllevaría una menor sensibilidad de la prueba de cribado.

En enero de 2013 se introdujo en muchos centros privados españoles el estudio de ADN fetal en sangre materna para el diagnóstico de alteraciones de los pares cromosómicos 13, 18 y 21 . En 1997 el descubrimiento de la presencia de ADN fetal circulante en sangre periférica materna (12), abrió un campo de investigación generando enormes expectativas acerca de la posibilidad de realizar diagnósticos prenatales sin la necesidad de procedimientos invasivos. Cuando surgieron los primeros estudios el fin era que este test llegara a sustituir a los procedimientos invasivos pero, al no llegarse a los niveles de sensibilidad y especificidad del $100 \%$, se empezó a hablar de los mismos como una herramienta de cribado (13), más que de diagnóstico.

La mayoría de los estudios se han centrado en población de alto riesgo seleccionada por el cribado combinado y/o marcadores ecográficos en el primer o segundo trimestre (14), por lo que tampoco queda claro si serían aplicables a la población general. En nuestro medio, podría realizarse a todas las gestantes con resultado de cribado combinado de primer trimestre y cribado de segundo trimestre de alto riesgo, lo que ahora supone un $74 \%$ de las indicaciones de amniocentesis. En la visita de las 12-14 semanas en la que informamos del riesgo, se les propondría la realización del estudio de ADN fetal, citándolas a resultados en dos semanas, todavía a tiempo de realizar estudio de cariotipo por medio de reacción en cadena de la polimerasa cuantitativa fluorescente (QF-PCR), disponible el resultado en 24-48 horas en los casos de test de ADN fetal positivo.

En el caso de pacientes con importantes marcadores ecográficos o malformaciones asociadas, el estudio de ADN fetal se desestimaría por no poder descartar éste otras anomalías cromosómicas que, aunque excepcionales, tienen gran expresividad ecográfica, además de que la sensibilidad para la detección de trisomía 13 y 18 se ha estimado en un $92 \%$ y un $96 \%$ respectivamente (15). Por ello, una duda que se nos plantea es cual es el peso de la translucencia nucal como para permitirnos el desestimar la amniocentesis en el caso de estudio de ADN fetal normal y TN mayor del P95.

Aunque la incorporación del estudio de ADN fetal al cribado del primer trimestre disminuiría dramáticamente la prevalencia de síndrome de Down en el segundo trimestre, el sonograma genético tendría su importancia en el asesoramiento de una prueba invasiva en pacientes con resultado no válido, hasta un 4,8\% (15), embarazos múltiples, y en los casos de resultados límite cuando los test no invasivos de diagnóstico prenatal den los resultados según la probabilidad de riesgo específico para cada paciente (16).

El cribado combinado de primer trimestre es el mejor método de tamizaje de síndrome de Down y debe ser ofertado a todas las gestantes, independientemente de su edad, como cribado universal. En un futuro próximo el estudio de ADN fetal en sangre materna deberá ser incorporado al cribado en pacientes de alto riesgo, como paso previo a la realización de una prueba invasiva. El alto coste actual de la prueba va a limitar su introducción en los sistemas públicos de salud, al menos para aplicar de forma universal a todas las embarazadas. Por ello se propone usar el cribado combinado para dividir a la población en tres grupos: muy alto riesgo, riesgo intermedio y riesgo bajo (17). A las pacientes del primer grupo les ofreceríamos de entrada una prueba invasiva, a las de bajo riesgo nada, y sería a las pacientes de riesgo intermedio las que se beneficiarían del estudio de ADN fetal. Se estima que con esta política se sometería al $25 \%$ de las gestantes a la prueba si el punto de corte considerado alto riesgo estuviera entre $1 / 11$ y $1 / 2500$ para detectar el $98 \%$ de las trisomías 13,18 y 21 (18). Es necesario que la administración pública y las sociedades científicas tengan en cuenta estos cambios en el Diagnóstico Prenatal para poder incorporarlos lo antes posible a nuestra práctica diaria. 


\section{CONCLUSIÓN}

El cribado combinado de primer trimestre ha demostrado una mayor tasa de detección para trisomía 21 que el cribado de segundo trimestre y/o la edad materna, además de que ha llevado a una importante reducción en el número de pruebas invasivas. En los próximos años la incorporación del estudio de ADN fetal mejorará la detección de aneuploidías con una drástica disminución de las pruebas invasivas por lo que se hace necesario la implantación de nuevos protocolos.

\section{REFERENCIAS}

1. Nicolaides K., Falcón O. La ecografía de las 11-13+6 semanas. Fetal Medicine Foundation, Londres 2004. Disponible en: http://www.fetalmedicine.com/fmf/ FMF-spanish.pdf.

2. Nicolaides KH, Azar G, Byrne D, Mansur C, Marks K. Fetal nuchal translucency: ultrasound screening for chromosomal defects in first trimester of pregnancy. BMJ 1992;304(6831):867-9.

3. Instrucción 01/1998 CatSalut. Implantación del diagnóstico prenatal de anomalías congénitas en Cataluña. Barcelona: Dirección General de Salud Pública; 1998. Generalitat de Catalunya. Departamento de Salut. Protocolo de Diagnóstico Prenatal de anomalías congénitas fetales en Cataluña. Barcelona: Dirección General de Salud Pública; 2008. Disponible en: http://www20.gencat.cat/docs/canalsalut/Home\%20 Canal\%20Salut/Professionals/Recursos/Protocols_i recomanacions/17_salut_de_la_mare_i_de_linfant/ documents/protocoldiagnosticprenatalbreu.pdf.

4. Grupo de Trabajo de la Sociedad Española de Ginecología y Obstetricia (SEGO). Guía de práctica clínica: Diagnóstico prenatal de los defectos congénitos. Cribado de anomalías congénitas. Diagn Prenat 2013;24(2):57-72.

5. Snijders RJ, Noble P, Sebire N, Souka A, Nicolaides $\mathrm{KH}$. UK multicentre project on assessment of risk of trisomy 21 by maternal age and fetal nuchal-translucency thickness at 10-14 weeks of gestation. Lancet 1998;352(9125):343-6.

6. Fortuny A, Gómez ML, Ortega MD, Montalvo J, Valero $\mathrm{J}$, Troyano J, et al. Propuesta de screening combinado de cromosomopatías en el primer trimestre de la gestación para todo el territorio nacional. Recomendaciones para la organización de un Servicio de Obstetricia y Ginecología. Documento SEGO 2005 Disponible en: http://www.aebm.org/documentos/ screening\%20gestante.pdf.
7. Manzanares S, Pineda A, Durán MD, López MS, GaIlo JL. Cribado de cromosomopatías fetales en España. Cambios hospitalarios en el periodo 2006-2011. Diagn Prenat 2013;24(1):3-10.

8. UK national screening committe (UK NSC). Department of Health. Fetal anomaly screening programme. Screening for Downs syndrome: UK NSC policy recommendations 2011-2014 Model of Best Practice. Disponible en: www.fetalanomaly.screening.nhs.uk

9. Gómez A, Abarca LJ, Ávila S, Villalón MC, García JM, Repollés M. Cribado combinado para la detección de trisomía 21 en el primer trimestre de la gestación. Impacto sobre la tasa de procedimientos invasivos de diagnóstico prenatal tras 5 años de implementación. Prog Obstet Ginecol 2012;55(4):173-80.

10. Tapia A. Estudio de coste-efectividad del cribado combinado del síndrome de Down en el primer trimestre de la gestación. Gestión y evaluación de costes sanitarios. Gestión y evaluación de costes sanitarios. Vol 12, monográfico 1. Enero-marzo 2011. Disponible en: http://www.fundacionsigno.com/archivos/ publicaciones/2011m\%2012\%201\%20p225.pdf.

11. Illescas T, Coronado PJ, Ortega MD, Soler P, Costa G, Montalvo J. Estudio descriptivo del cribado de cromosomopatías en el primer trimestre de la gestación en el Hospital Clínico San Carlos de Madrid, España. Rev Chil Obstet Ginecol 2011;76(5):318-24.

12. Lo YM, Corbetta N, Chamberlein PF, Rai V, Sargent IL, Redman CW, et al. Presence of fetal DNA in maternal plasma and serum. Lancet 1997;350(9076):485-7.

13. Rodríguez M, Bustamante A, Perlado S, Trujillo MJ, Díaz J, Plaza J, et al. Diagnóstico prenatal no invasivo: presente y futuro de mano de las nuevas tecnologías. Diagn Prenat 2012;23(2):67-75.

14. Nicolaides K, Syngelaki A, Ashoor G, Birdir C, Touzet G. Noninvasive prenatal testing for fetal trisomies in a routinely screened first-trimester population. Am J Obstet Gynecol 2012;207:374,e1-6.

15. Gil M, Quezada M, Bregnant B, Ferraro M, Nicolaides $\mathrm{K}$. Implementation of maternal blood cell-free DNA testing in early screening for aneuploidies. Ultrasound Obstet Gynecol 2013;42:34-40.

16. Odibo A, Ghidini A. Role of the second-trimester "genetic sonogram" for Down Syndrome screen in the era of first-trimester screening and noninvasive prenatal testing. Prenat Diagn 2014;34(6):511-517.

17. Gratacós E, Nicolaides K. Clinical perspective of cellfree DNA testing for fetal aneuploidies. Fetal Diagn Ther 2014;35(3):151-5.

18. Gil MM, Akolekar R, Quezada MS, Breggant B, Nicolaides $\mathrm{KH}$. Analysis of cell-free DNA in maternal blood in screening for aneuploidies: meta-analysis. Fetal Diagn Ther 2014; 35(3):156-73. 